\title{
Ras Dependent Paracrine Secretion of Osteopontin by NfI+/- Osteoblasts Promote Osteoclast Activation in a Neurofibromatosis Type I Murine Model
}

HUIJIE LI, YALING LIU, QI ZHANG, YONGMIN JING, SHI CHEN, ZHAOHUI SONG, JINCHENG YAN, YAN LI, XIAOHUA WU, XIANGHONG ZHANG, YINGZE ZHANG, JAMIE CASE, MENGGANG YU, DAVID A. INGRAM, AND FENG-CHUN YANG

Herman B Wells Center for Pediatric Research [H.L., Y.L., Q.Z., Y.J., S.C., Z.S., J.Y., Y.L., X.W., J.C., D.A.I., F.-C.Y.], Departments of Pediatrics [H.L., Y.L., Q.Z., Y.J., S.C., Z.S., J.Y., Y.L., X.W., J.C., D.A.I., F.-C.Y.], Biostatics [M.Y.], Biochemistry and Molecular Biology [D.A.I.], Anatomy and Cell Biology [F.-C.Y.], Indiana University School of Medicine, Indianapolis, Indiana 46202; Department of Orthopedic [H.L., Y.L., Q.Z., Y.J., Z.S., J.Y., X.Z., Y.Z.], The $3^{\text {rd }}$ Hospital, Hebei Medical University, Hebei, China 050051

\begin{abstract}
Neurofibromatosis type 1 (NF1) is a pandemic genetic disorder characterized by malignant and nonmalignant manifestations, including skeletal abnormalities, such as osteoporosis, scoliosis, short stature, and pseudarthrosis. Recent studies in genetically inbred mice and from human patients with NF1 have identified multiple gains in osteoclast (OCL) functions both in vitro and in vivo. Given that osteoblasts secrete cytokines that promote OCL maturation/activation, we sought to identify whether haploinsufficiency of $N f 1(N f 1+/-)$ osteoblasts and their precursors secrete cytokines that have a central role in this process. Osteoblast conditioned media (OBCM) from $\mathrm{Nfl}+/-$ osteoblasts promoted OCL migration and bone resorption compared with WT OBCM. Osteopontin (OPN), a matrix protein found in mineralized tissues and pivotal in modulating OCL functions, was present in increased concentrations in $\mathrm{NfI}+\mathrm{/}-$ osteoblasts. Addition of OPN neutralizing antibody to $\mathrm{NfI} / \mathrm{/}$ OBCM diminished the gain in bioactivity on OCL functions, including OCL migration and bone resorption. Our study identifies an important paracrine loop whereby elevated secretion of OPN by osteoblasts activate $N f I+/-$ OCLs that already have an intrinsic propensity for bone resorption leading to osteopenia and osteoporosis. (Pediatr Res 65: 613-618, 2009)
\end{abstract}

$\mathrm{N}$ eurofibromatosis type 1 (NF1) is a common, autosomal dominant disorder caused by mutations in the NFI tumor suppressor gene that encodes the protein neurofibromin. $N F 1$ functions as a GTPase in mesothelial-derived tissues including blood cells, fibroblasts, and osteoprogenitor cells that are relevant to skeletal development (1-3). Patients with NF1 have a range of malignant and nonmalignant manifestations. Although many laboratories have recently identified potential molecular targets for the treatment of the neoplasms associated with NF1, significantly less effort has been focused on understanding the molecular mechanisms underlying the pathogenesis of many of the nonmalignant manifestations of NF1, particularly the skeletal manifestations that cumulatively affect up to $50 \%$ of all patients with NF1 (4-6). Recent clinical studies have found that individuals with NF1 are at

Received September 19, 2008; accepted January 29, 2009.

Correspondence: Feng-Chun Yang, M.D., Ph.D., Indiana University School of Medicine, Cancer, Research Institute, 1044 W. Walnut Street, R4/427, Indianapolis, IN 46202; e-mail: fyang@iupui.edu

Supported by March of Dimes Foundation \#6-FY08-246 (F.C.Y.)

The first two authors contributed equally to this work. significant risk for both generalized and focal skeletal abnormalities, including osteoporosis and osteopenia $(7,8)$.

Osteoclasts (OCLs) are terminally differentiated multinucleated giant cells with a principal bone resorptive activity, and they play a central role in the regulation of bone mass (9). Bone resorption seems to proceed by the intricate coordination of the processes of attachment to bone, polarized secretion of acid and proteases, and active motility of OCLs along the bone surface. As OCLs crawl over bone surfaces, they require rapid attachment and release from the extracellular matrix. Recently, we reported that $N f 1$ haploinsufficient $(N f 1+/-)$ OCLs have an increased bone resorption compared with WT control OCLs (10).

OPN is a secreted, noncollagenous, sialic acid-rich phosphoglycoprotein that acts both as a chemokine and cytokine and is associated with cell adhesion, migration, extracellular matrix invasion, inhibition of calcification, and cell proliferation (11-13). OPN has been reported to be involved in bone resorption, angiogenesis, wound healing, and tissue injuries following binding with type I collagen, fibronectin, and osteocalcin $(14,15)$. In bone, it is predominantly expressed in osteoblasts and in terminally differentiated chondrocytes at the chondroosseous border (16). OPN-deficient mice are resistant to ovariectomy-induced bone resorption (17).

Yu et al. (3) have shown that heterozygous loss of $\mathrm{NfI}$ enhances OPN expression in bone marrow-inducible osteoprogenitors and cortical bone. These studies suggest the potential for an interaction between OPN and gain in functions in NfI haploinsufficient OCLs, though no functional evaluation has been reported. In this study, we investigated the impact of osteoblasts on the $\mathrm{NfI}+/-$ OCL cellular functions. Our study indicates that OPN in $N f 1+/-$ osteoblast conditioned medium $(\mathrm{OBCM})$ contributes to the pathologic function of $\mathrm{Nfl} \pm$ OCLs as measured by OCL migration, adhesion, and bone resorption. Further, introduction of the GAP-related domains (GRD)

Abbreviations: BMMNCs, bone marrow mononuclear cells; Erk, extracellular signal-regulated kinase; GRD, GAP related domain; NF1, neurofibromatosis type 1; $N f 1+/-$, haploinsufficiency of $N f 1$; OBCM, osteoblast conditional media; OCL, osteoclast; OPN, osteopontin; TRAP, tartrate resistant acid phosphatase 
of NF1 via a recombinant retrovirus that encodes the region of NF1 required for Ras signaling corrects this pathologic secretion of OPN, indicating a Ras dependent secretory effect. Collectively, these studies identify a Ras dependent paracrine osteoblast-OCL interaction that could have implications to osteoporosis in children and adults with NF1.

\section{MATERIALS AND METHODS}

Animal. $\mathrm{Nf1}+/-$ mice were obtained from Tyler Jacks at the Massachusetts Institute of Technology (Cambridge, MA) in a C57BL/6J.129 background and backcrossed for 13 generations into a C57BL/6J strain (10).

Preparation of primary calvarial osteoblasts and osteoblast conditional media. The osteoblastic cells were isolated from the calvariae of 3-d-old WT and $\mathrm{NfI} / \mathrm{/}-$ mice. The calvariae were digested in $\alpha$-minimal essential medium ( $\alpha$-MEM; Invitrogen, Carlsbad, CA) containing $0.02 \%$ type I collagenase, $0.05 \%$ trypsin, and $0.53 \mathrm{mM}$ ethylene-diamine-tetra-acetic acid for 20 $\mathrm{min}$. The digestion procedure was repeated six times, and the cells isolated by the last three digestions were combined as an osteoblastic cell population. The osteoblastic cells were cultured in $\alpha$-MEM containing $10 \%$ fetal bovine serum (FBS, Biomeda, Chess Drive Foster City, CA), $5 \mathrm{mM} \beta$-glycerophosphate, and $100 \mu \mathrm{g} / \mathrm{mL}$ ascorbic acid. The staining of alkaline phosphatase was used for the confirmation of osteoblast culture (18). OBCM was collected from each osteoblast cell culture line after 24-h starvation with $\alpha$-MEM containing $0.5 \% \mathrm{BSA}$ at $37^{\circ} \mathrm{C}, 5 \% \mathrm{CO}_{2}$ or from the osteoblast differentiated from mesenchymal stem/progenitor cell culture as described previously (18). The supernatants were then collected and stored in aliquots at $-80^{\circ} \mathrm{C}$.

ELISA. Quantity of OPN concentration was performed by ELISA using Quantikine M according to the manufacture's protocol (R\&D Systems, Inc., Minneapolis, MN).

Generation of murine OCLs and tartrate resistant acid phosphatase staining. Mouse OCLs were generated in vitro using mouse bone marrow mononuclear cells (BMMNCs) as described previously (10). BMMNCs from 6- to 8-wk-old $\mathrm{NfI}+/-$ and WT mice were cultured in $\alpha$-MEM supplemented with $10 \% \mathrm{FBS}$ in the presence of murine recombinant macrophage-colony stimulating factor (M-CSF, $30 \mathrm{ng} / \mathrm{mL}$, Peprotech, Rocky Hills, NC) for $3 \mathrm{~d}$. On d 4, cell culture media was switched to $\alpha$-MEM supplemented with $10 \%$ FBS, M-CSF (30 ng/mL), murine recombinant RANKL (30 ng/mL), and OPN (R\&D Systems, Minneapolis, MN) for another $3 \mathrm{~d}$. In some of the experiments, the OCLs $\left(1 \times 10^{5} /\right.$ well in 96 wells plate $)$ were cultured in the presence of OBCM $(200 \mu \mathrm{L} /$ well) and/or neutralizing antibody (R\&D system, 2 $\mu \mathrm{g} / \mathrm{mL}$ ). To identify OCLs at the end of the culture period, adherent cells were fixed with $10 \%$ formaldehyde in PBS, treated with ethanol-acetone (50:50), and stained for tartrate resistant acid phosphatase (TRAP) as described previously (10) and then visualized with a Nikon TE2000-S microscope (Nikon Inc., Melville, NY). Images were taken by a QImaging camera and QCapture-Pro software (Fryer Company Inc., Cincinnati, OH). Multinucleated $\mathrm{TRAP}^{+}$cells containing more than three nuclei were scored as mature OCLs. The area of multinucleated, TRAP ${ }^{+}$OCLs ( $\%$ of field) was calculated by measuring with Metamorph offline software.

Bone resorption assay. OCLs were gently scraped as described previously (10). Single-cell suspensions of purified OCLs $\left(2 \times 10^{4} /\right.$ well $)$ were seeded on prewet dentine slices (ALPCO Diagnostic, Windham, $\mathrm{NH}$ ) and incubated at $37^{\circ} \mathrm{C}, 5 \% \mathrm{CO}_{2}$, with/without OPN $(5 \mu \mathrm{g} / \mathrm{mL})$, in the presence of M-CSF and RANKL or in the presence of OBCM (200 $\mu \mathrm{L} /$ well), and/or OPN neutralizing antibody $(2 \mu \mathrm{g} / \mathrm{mL})$. The number of resorptive areas or "pits" per low-power field on each bone slice was counted using reflective light microscopy after staining with $1 \%$ toluidine blue in $0.5 \%$ sodium tetraborate solution. The area of pit (\% of field) was measured with Metamorph offline software.

Migration assay. Transwell assays were used to measure the OCL migration induced by M-CSF (30 ng/mL), OPN, or OBCM $(600 \mu \mathrm{L})$, and/or OPN antibody $(2 \mu \mathrm{g} / \mathrm{mL})$ using a $8-\mu \mathrm{m}$ pore size transwell system (Costar, Boston, MA) as previously described with minor modifications (10).

Osteoclast adhesion assay. A single-cell suspension of OCL precursors $\left(1 \times 10^{4}\right.$ cells/well $)$ were placed into 96 -well plates coated with $5 \mu \mathrm{g} / \mathrm{mL}$ OPN or with PBS as previously described (10). After $20 \mathrm{~min}$, nonattached cells were gently washed away, and attached cells were fixed and counted.

Evaluation of biochemical activities. Phosphorylation of Akt and extracellular signal-regulated kinase (Erk) were determined by Western blotting using phosphospecific antibodies (New England Biolabs, Beverly, MA) for Akt and Erk as previously described (10). To measure OPN expression, osteoblast generated from WT and $\mathrm{NfI}+/-$ mice were cultured as described above. After $24 \mathrm{~h}$ starvation in 0.5\% BSA in DMEM, cells were lysed for Western blotting. All signals were visualized using ECL chemiluminescence substrate (Amersham Pharmacia Biotech, Piscataway, NJ). $\beta$-actin was used as an internal control. The intensity of the bands on the blots was measured with Alpha Innotech Software.

Generation of recombinant retroviral plasmids and retroviral transduction. Previously developed recombinant retrovirus constructs were used in these studies: a virus expressing the full-length NF1 GRD and pac (MSCVNF1 GRD-pac) and a virus expressing the selectable marker gene alone (MSCV-pac) (19). Retroviral transduction was performed as previously described $(18,19)$. After the transduction, OBL differentiation media were added into OBL progenitor cultures for $7 \mathrm{~d}$, and the conditional media were collected as described earlier.

Statistics. Repeated measurement ANOVA and paired $t$ test were used to evaluate statistical differences between the results obtained with WT and $N f 1+/-$ cultures or mice. Multiple comparisons were adjusted using Bonferroni correction. The $p$ values $<0.05$ were considered significant.

\section{RESULTS}

Conditioned media from $\mathrm{Nf1+/-} \mathrm{osteoblasts} \mathrm{promotes} \mathrm{the}$ OCL formation. Osteoblasts have been shown to have the potential of promoting OCL maturation (20). To evaluate whether the haploinsufficient loss of $N f 1$ in osteoblasts alters OCL maturation, OBCM from WT or $N f 1+/-$ cultures was collected and used for the OCL formation from the BMMNCs. As shown in Fig. $1 A-B$, OBCM from both WT and $N f 1+/-$ osteoblast cultures has the capacity to promote WT or $\mathrm{Nf1}+/ \mathrm{-}$ OCL formation. Interestingly, conditional media from $\mathrm{NfI}+/$ - osteoblast culture has a greater bioactivity on OCL

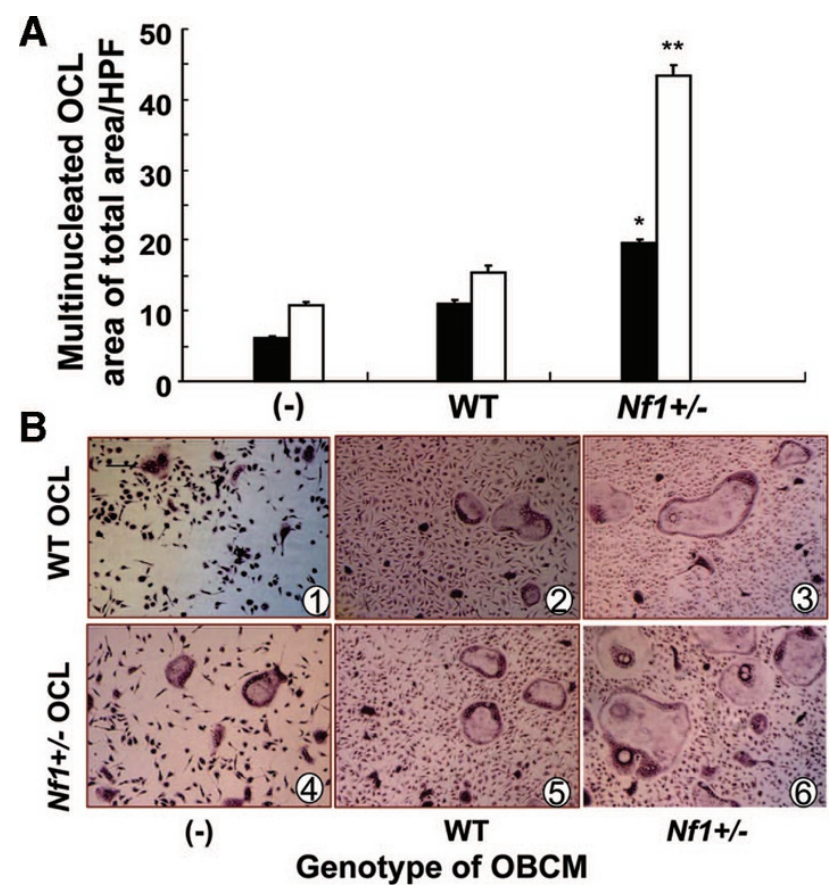

Figure 1. The promotion effect of $N f 1+/-$ OBCM on OCL formation is significant for both WT and $N f 1+/-$ cell types. (A) WT and $N f 1+/-$ OBCM was added to the OCL culture (half OBCM and half $10 \%$ FBS in $\alpha$-MEM), and after $7 \mathrm{~d}$ of culture, the area of TRAP $^{+}$multinucleated cells were counted. Data represent the mean \pm SEM of six high power field $(\mathrm{HPF}, 100 \times)$ per condition. Data were collected from three individual mice for each genotype and three independent experiments were performed. The estimated mean difference in the area of multinucleated WT OCLs ( $\square$ ) induced by $\mathrm{NfI} / \mathrm{/}$ OBCM or WT OBCM is $8.3(* p<0.0001)$, whereas the estimated mean difference in the area of $N f 1+/-$ multinucleated OCLs $(\square)$ induced by $N f 1+/-$ OBCM and WT OBCM is $28(* * p<0.0001)$. The difference of 28 in the area of $\mathrm{NfI} / \mathrm{/}$ multinucleated OCLs is significantly higher than the difference of 8.3 in the area of WT multinucleated OCLs $(p<0.0001)$. (B) A representative photomicrograph was shown. Magnification bar $=20 \mu \mathrm{m}$. 

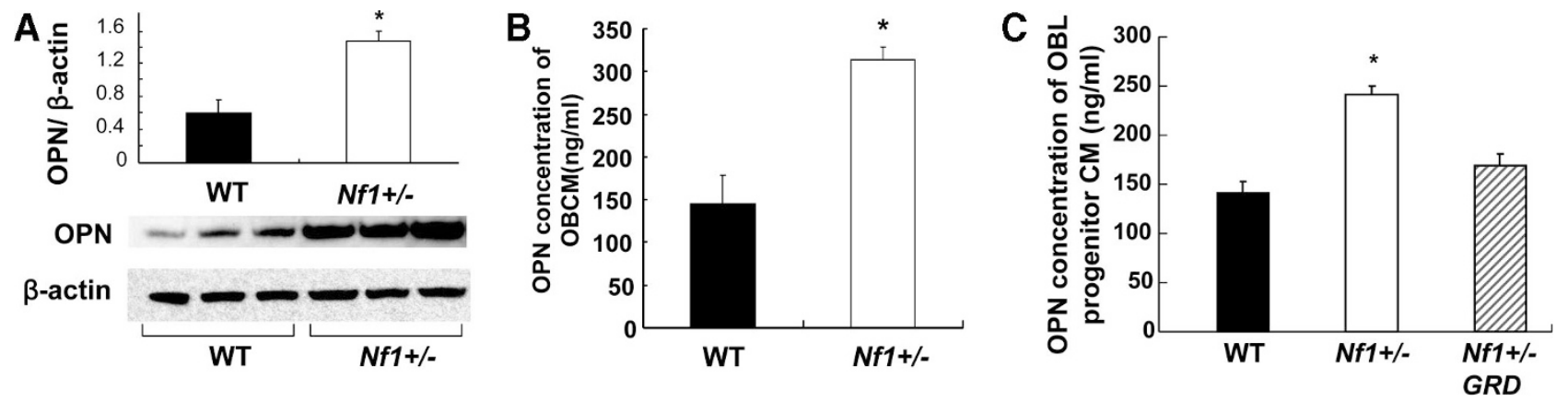

Figure 2. $N f 1+/-$ OBCM contains higher OPN expression. (A) The expression of OPN in WT and $N f 1+/-$ osteoblasts was examined by Western blot. Data were obtained from three independent osteoblast lines derived from three mice in each experimental group. * $p<0.05$ for comparing WT $v s$. Nf1 $+/-$ osteoblasts. (B) OPN level in OBCM was measured by ELISA. Experiment conducted on three independent occasions qualitatively. * $p<0.05$ for comparing WT $v s . N f 1+/-$ OBCM. (C) OPN level in OBCM was measured following osteoblast differentiation for $7 \mathrm{~d}$ using OBL progenitors transduced with NF1 GRD or MSCV-pac control. $* p<0.01$ for comparing $N f 1+/-$ pac with WT pac or $N f 1+/-$ GRD OBCM. Data represents one of three independent results.

maturation in both WT and NfI+/- OCLs compared with WT OBCM. This data implies that $N f 1+/-$ OBCM contains soluble factor(s) that influences the maturation of OCLs.

Increased level of OPN in Nf1+/-OBCM and in the serum of $N f 1+/-$ mice and re-expression of NF1 GRD into Nf1+/- osteoblast progenitors normalizes the OPN secretion. It has previously been reported that the loss of $N f 1$ in osteoblasts alters the OPN mRNA expression in vitro by RTPCR $(3,21)$. We qualitatively and quantitatively measured OPN expression by western blot and ELISA. Consistent with previous reports, a dramatic elevation of OPN expression in $N f 1+/-$ osteoblasts was observed compared with that in WT cells when measured by Western blot (Fig. 2A). Furthermore, a significantly higher concentration of OPN was detected in the $N f 1+/-$ OBCM by ELISA compared with that of WT OBCM (Fig. 2B). Similarly, a significantly higher concentration of OPN was also observed in serum collected from $N f 1+/-$ mice compared with that of WT mice ( $308 \pm 23$ vs. $225 \pm 10.7$, respectively, $n=4$ ). To determine whether increased OPN level in the OBCM in $N f 1+/-$ culture is directly related to altered p21ras, WT, and NfI+/- OBL progenitor cells were transduced with a recombinant retrovirus encoding full length NF1 GRD and a selectable marker, pac, selected in puromycin, and subjected to culture for further osteoblast differentiation (18). In contrast to OBCM collected from $\mathrm{NfI+/-}$ OBL expressing MSCV-pac alone, expression of NF1 GRD in $\mathrm{NfI}+/-\mathrm{OBL}$ progenitor cells normalized OPN secretion (Fig. $2 C$ ). Thus, the increased level of OPN in OBCM is directly linked to loss of neurofibromin and alteration of Ras activity.

OPN induces OCL formation and increases $N f 1+/-O C L$ adhesion and migration. Given the gain in OCL functions in vivo in $\mathrm{Nfl}+/-$ mice (10), we hypothesized an association between the in vivo phenotypes and increased level of OPN. To test the hypothesis, we performed OCL formation assays in response to a range of concentrations of OPN in the presence of M-CSF and RANKL. This was accomplished by using an established methodology (10) to differentiate bone marrow cells in M-CSF for $4 \mathrm{~d}$ in vitro which induces a enriched population of macrophages that have lost their clonogenic potential. Both WT and $N f I+/-$ cultures show a significantly increasing pattern of OCL formation in response to different dosages of OPN. A consistent larger area of multineucleated
OCLs was observed from $N f 1+/-$ BMMNC culture induced by a range of dosages of OPN compared with that of WT cultures (Fig. 3A).

Given that OPN is a ligand for several cell surface adhesion receptors (13) and that OCL adhesion and migration are important processes during OCL bone resorption, we next examined the impact of OPN on OCL adhesion in both $\mathrm{Nfl}+/-$ and WT OCLs. As shown in Fig. 3B, a 1.6-fold increase in the number of adherent cells was observed in OPN stimulated WT OCLs when compared with PBS treated WT OCLs (Fig. 3B). In contrast, a 3.5-fold increase in adherent cells was observed in OPN treated $N f 1+/-$ cultures compared with PBS treated $N f 1+/-$ OCLs. OPN has been known to regulate OCL motility (22), and we further examined OPNmediated OCL migration using the transwell assay. $\mathrm{Nfl}+/-$ OCLs demonstrated significantly higher OCL migration when compared with WT cells under basal unstimulated condition. M-CSF induced a significant increase in migration in both WT and $N f 1+/-$ OCLs over baseline. Importantly, addition of OPN to M-CSF gave rise to a greatest migration in $\mathrm{NfI} / \mathrm{l}$ OCLs, although an increased migration was also observed in WT cells compared with M-CSF alone (Fig. $3 C$ and $D$ ).

OPN preferentially promotes bone resorption of $\mathrm{NfI}+/-$ OCLs. A key role of OCLs is to resorb bone in vivo. The process of bone resorption can be measured by the "pit formation" assay. Using the established pit formation assay (10), a consistent increase in the M-CSF-mediated bone resorption and RANKL-mediated bone resorption was observed in $N f 1+/-$ OCLs (Fig. $4 A$ and $B$ ). Importantly, OPN induced a 4 -fold increase in OCL resorption in $\mathrm{NfI} / \mathrm{/}$ - cultures compared with cultures lacking OPN.

Neutralizing antibody for OPN inhibited bioactivity of $N f 1+/-$ OBCM. To confirm that the gain in $N f 1+/-$ OBCM mediated OCL functions are OPN related, we applied neutralizing antibody for OPN into the culture, and repeated OCL formation, migration, and pit formation. Consistently, a significant increase in multinucleated OCL formation was observed in $\mathrm{Nfl}+/ \mathrm{-OCL}$ culture in the presence of $\mathrm{Nfl}+/-$ OBCM compared with that in the WT OCL culture (Fig. 5A and $B$ ). Further, addition of OPN neutralizing antibody to the $N f 1+/-$ OBCM significantly reduced OCL formation (Fig. $5 A$ and $B$ ). In addition, anti-OPN minimized OCL migration in 

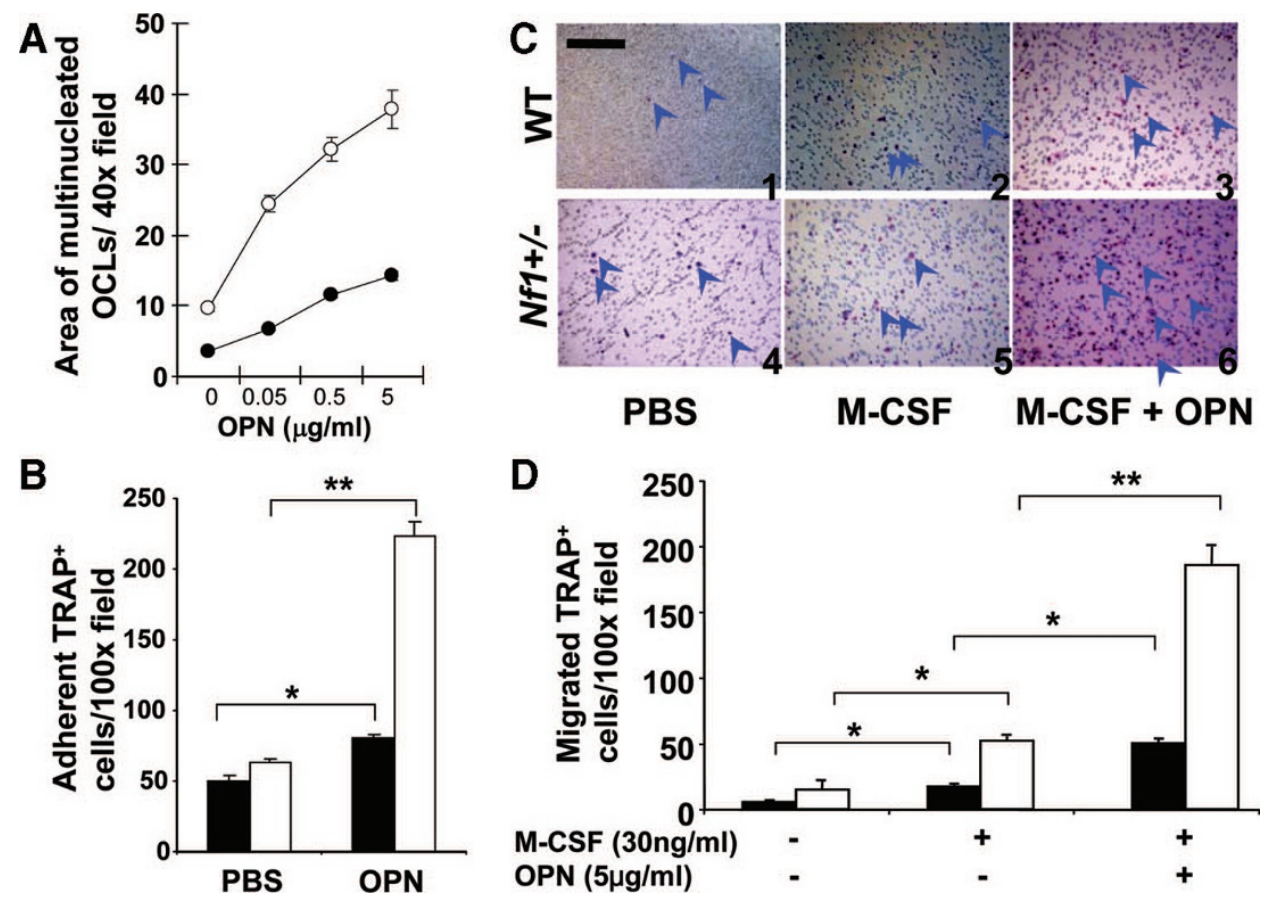

Figure 3. OPN augments OCL formation in $N f 1+/-$ culture and increases adhesion and migration in WT and $N f 1+/-$ OCLs. (A) Quantitative evaluation of the area of multinucleated TRAP ${ }^{+}$OCLs following a range of concentrations of OPN stimulation in in vitro culture. Repeated measurement regression analysis was used where positive dosages of OPN were $\log _{10}$-transformed. Both WT and $\mathrm{Nfl}+/$ - cultures show a significantly increasing pattern of OCL formation. An estimated slope for WT OCL formation $(-\bullet-)$ is $2.94(p<0.0001)$. This can be interpreted as for every 10 fold increase of dosage, the increase in the area of multinucleated OCLs is 2.94. In contrast, an estimated slope for $N f 1+/-$ OCL formation $(-\circ-)$ is $7.70(p<0.0001)$. This can be interpreted as: for every 10 fold increase of dosage, the increase in the area of multinucleated OCLs is 7.70. A significantly different of the slopes between WT and Nf1+/- multinucleated OCLs was observed $(p<0.0001)$. $(n=5)(B)$ Adhesion of OCL precursors was examined. Data represent mean \pm SEM from eight fields per condition and a summary of three independent experiments is shown. $y$ axis shows percent increase in adherent OCLs over basal level. * $p<0.01$ for WT OCL adhesion with $v s$. without OPN. **p $<0.001$ for $N f 1+/-$ OCL adhesion with $v s$. without OPN. (C) OCL migration was evaluated by the transwell assay. Representative photomicrographs from four independent experiments are shown. Magnification bar $=20 \mu \mathrm{m}$. $(D)$ Quantitative evaluation of migration in response to media alone, M-CSF, or OPN with M-CSF. Data represent mean \pm SEM from 10 fields per condition. Experiments were conducted in triplicates and four independent

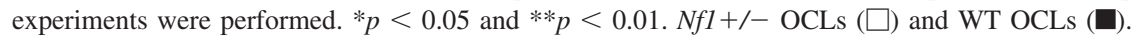
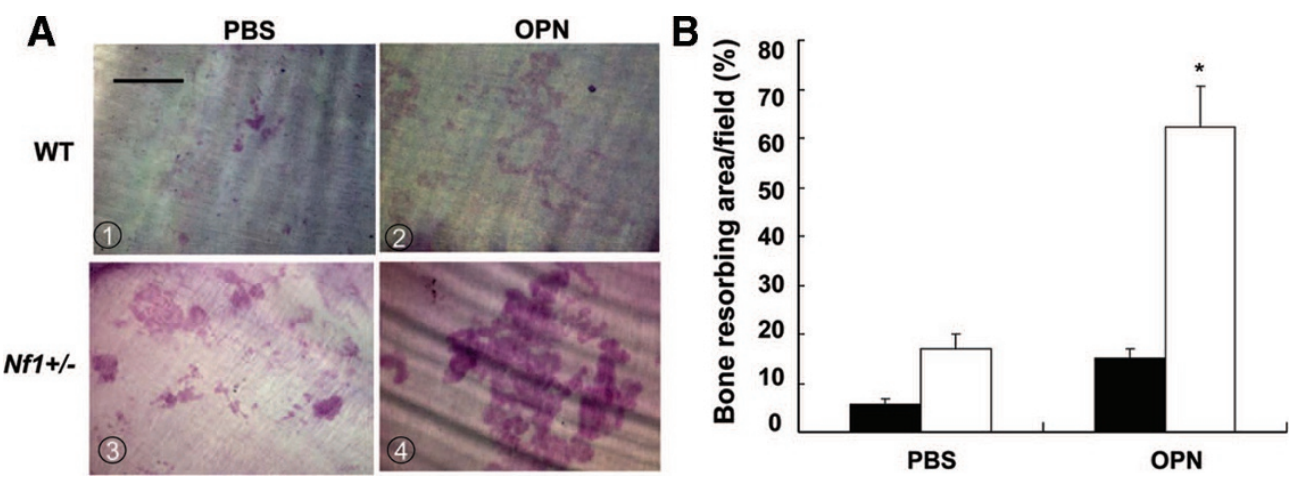

Figure 4. OPN preferentially promotes
bone resorption of $N f 1+/-$ OCLs. $(A)$
Representative photomicrographs of
bone resorption from WT or $N f 1+/-$
culture in the presence of M-CSF plus
RANKL, or in addition of OPN $(5 \mu \mathrm{g} /$
$\mathrm{mL})$. Magnification bar $=20 \mu \mathrm{m} .(B)$
Quantitative evaluation of bone resorp-
tion. Data represent the mean \pm SEM of
six fields per genotype. Each condition
was done in triplicates and four indepen-
dent experiments were performed. $* p<$
0.01 for $N f 1+/-(\square) v s$. WT $(\square)$.

both WT and $N f 1+/-$ OCL cultures mediated by $N f 1+/-$ OBCM as measured by the transwell assays (Fig. 5C). Finally, neutralizing OPN antibody diminished both WT and $N f 1+/-$ OCL pit formation associated with OBCM (Fig. 5D and $E$ ). Taken together, these results indicate that OPN in $N f 1+/-$ OBCM significantly contributes to the gain in $\mathrm{Nfl}+/-$ OBCM mediated OCL functions.

Nf1+/- OCLs have hyperactive Erk and Akt phosphorylation mediated by OPN. We have previously reported that the loss of NfI in OCLs leads to increased activity of Ras/ MAPK and Phosphoinositide 3-kinase (PI3-K) pathways (10). Other groups have also shown that OPN up-regulated the PI3-K and
MAPK pathways $(23,24)$. We next evaluated phosphorylation of Erk and Akt following OPN stimulation. Although we do not see a dramatic up-regulation of Erk or Akt phosphorylation in WT OCL induced by OPN, a marked increase in the Erk and Akt phosphorylation was observed in $\mathrm{Nfl}+/-$ OCLs after OPN stimulation (Fig. $6 A$ and $B$ ). To confirm that the PI3-K and MAPK pathways are important for the gain in OPN mediated $N f 1+/-$ OCL functions, PD98059, a MAPK inhibitor, and Ly294002, a PI3-K inhibitor, were applied to the cultures, and OPN-mediated OCL migration was repeated. Both PD98059 and Ly294002 were sufficient to block the

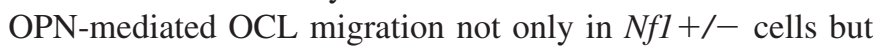



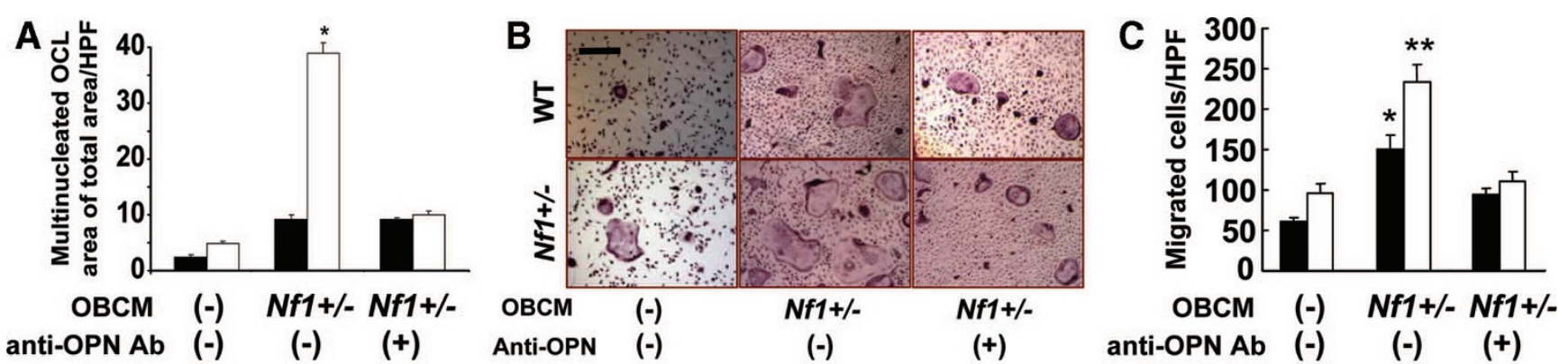

anti-OPN Ab (-) (-)

(+)

Anti-OPN

$(-)$

$(-)$

(+)

anti-OPN Ab (-)

$(-)$

(+)

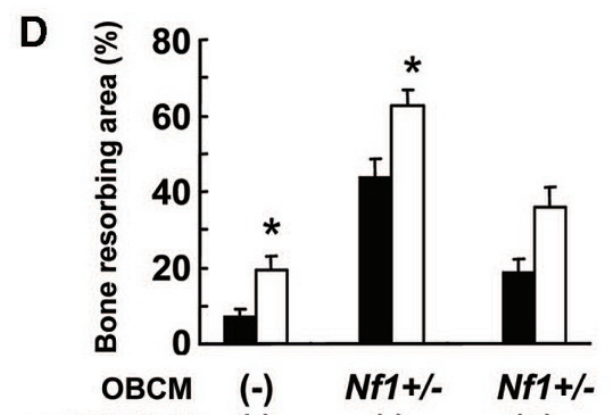

anti-OPN Ab (-)

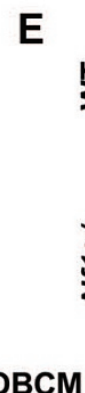

$(+)$

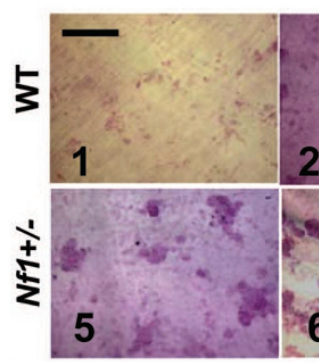

$(-)$

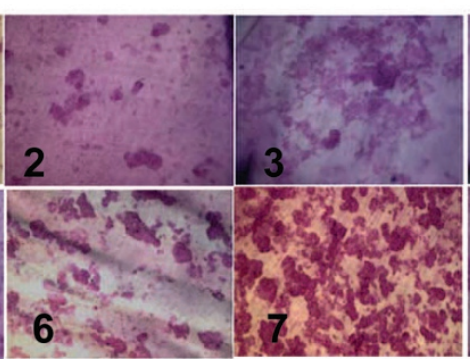

WT

$(-)$

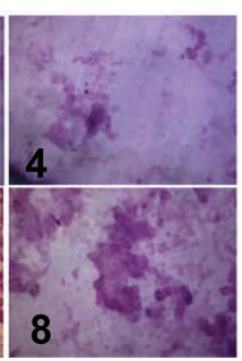

Nf1+/-

$(+)$

Figure 5. Neutralizing antibody for OPN inhibited bioactivity of $N f 1+/-$ OBCM. (A) Neutralizing antibody for OPN (5 $\mu \mathrm{g} / \mathrm{mL})$ was applied to the culture, repeated OCL formation was performed. The OCL formation was assessed by scoring the area of multinucleated TRAP + cells per HPF. * $p<0.01$ for comparing

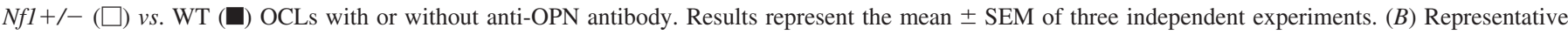
photomicrographs of the indicated genotypes are shown. Magnification bar $=20 \mu \mathrm{m}$. $(C)$. Neutralizing antibody for OPN was applied to the OBCM and migration was performed. $y$ axis indicates the number of migrated OCLs (HPF, 100 $\times$ ). ${ }^{*} p<0.01$ for comparing WT (ם) OCLs migration with or without anti-OPN antibody. $* * p<0.01$ for comparing $N f 1+/-(\square) v s$. WT (ם) OCLs with or without anti-OPN antibody. Results represent the mean \pm SEM of three independent experiments. $(D)$ Neutralizing antibody for OPN was applied to the OBCM and bone resorption assays were performed. $* p<0.01$ for comparing

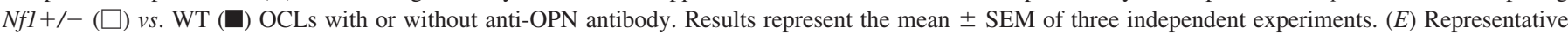
photomicrographs of the indicated genotypes are shown. Magnification bar $=20 \mu \mathrm{m}$. All the experiments were performed in triplicates.
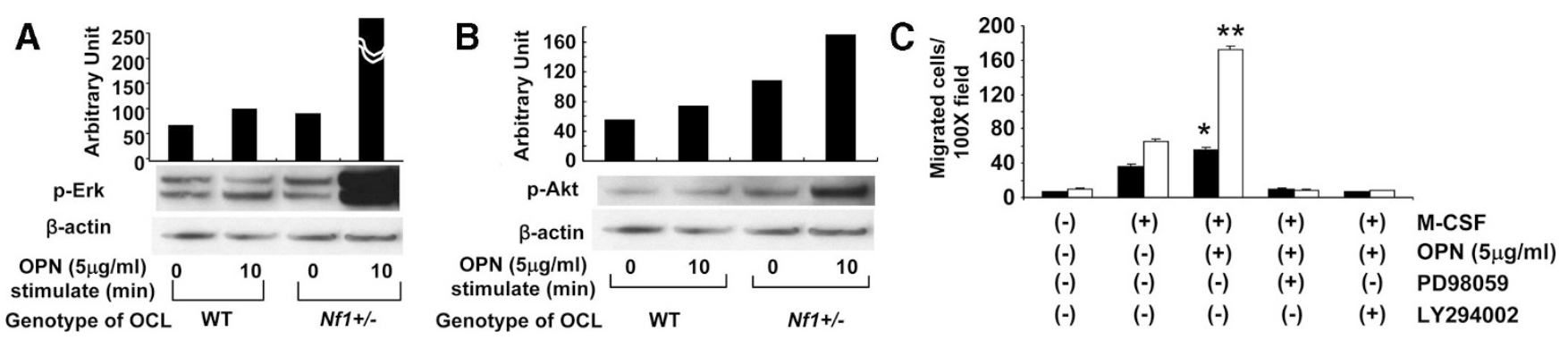

Figure 6. $N f 1+/-$ OCLs have hyperactive Erk and Akt phosphorylation mediated by OPN. $(A-B)$ Representative photomicrographs of Western blot of the indicated genotypes are shown. Experiment conducted on four independent occasions. (C) PD98059 and LY294002 inhibited OPN mediated OCL migration. PD98059 and LY294002 were applied to the lower chambers containing OPN and M-CSF and migration assays were performed. * $p<0.01$ for comparing the

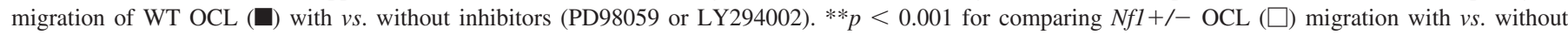
inhibitors (PD98059 or LY294002). Experiments were done in triplicates and results represent the mean \pm SEM of three independent experiments.

also in WT cells (Fig. 6C). These studies imply an association between the gain in OPN-mediated OCL migration and the PI3-K and MAPK pathways in NfI+/- OCLs.

\section{DISCUSSION}

NF1 is known as one of the most common genetic diseases in human with a predisposition to cancer. Despite NF1 being considered a neurocristopathy, nonmalignant manifestations of the disease contribute significantly to disease morbidity. From 30 to $50 \%$ of patients with NF1 have both generalized and focal skeletal abnormalities. Multiple recent reports have provided evidence that greater than $30 \%$ of patients with NF1 develop osteopenia and osteoporosis. Osteoporosis is an emerging phenotype observed even in young individuals, and consequently, patients with NF1 are even at a greater risk than the general population $(6,7)$. We recently provided evidence that $N f 1+/-$ in both humans and mice (NfI), respectively, results in increased OCL differentiation and functions, including migration, adhesion, and bone resorption (10). However, the paracrine interactions between the mesenchymal elements of bone and the hematopoietic OCLs are currently in its infancy. In this study, we investigated the impact of OPN on the functions of OCLs, including migration, adhesion, and bone resorption.

OPN is a secreted phosphoglycoprotein that has been linked to tumor progression and survival in several solid tumors (25). 
It also has been known that OPN is a positive regulator for osteoclastogenesis (22). El-Tanani et al. (26) reported an association of Ras with OPN expression in malignant cells. In this study, we observed an increased OPN expression in $\mathrm{Nfl}+/-$ osteoblasts and an increased serum level of OPN in $N f 1+/-$ mice. It has been established that loss of NF1, a GTPase for p21Ras, results in an activation of Ras in a number of lineages $(3,10,18,27)$. The increase in OPN expression in $\mathrm{Nfl}+/-$ mice may be a result of the hyper Ras activity in osteoblasts. Further, we found that haploinsufficient loss of $N f 1$ in OCLs leads to hypersensitivity to OPN, which leads to the $\mathrm{NfI}+/$ - gain-of-functional phenotype observed in vitro, including adhesion, migration, and bone resorption. Importantly, neutralizing antibody for OPN is sufficient to minimize the gain in $N f 1+/-$ OCL functions mediated by the conditioned media collected from $N f I+/-$ osteoblast cultures. These findings imply that inhibiting OPN may be a potential way for the treatment of osteoporosis in the NF1 population.

The Ras/Mek/Erk and PI3-K signaling cascade are critical in physiologic OCL function $(10,28)$. It has been reported that the Ras/Mek/Erk and PI3-K signaling pathways are hyperactivated in several disease models, including Paget's disease, bone metastasis, wear particle-induced osteolysis after arthroplasty, and NF1 $(10,18,29)$. Congenital disorders bearing phenotypic overlap with NF1, including Noonan syndrome, Noonan-like/multiple giant cell lesion syndrome, LEOPARD syndrome, Costello syndrome, and Cardio-facio-cutaneous syndrome, carry germline mutations within key genes contributing to hyperactivation of the Ras-Raf-Mek-Erk signaling cascade. It is being increasingly recognized that individuals with many of these disorders are predisposed to osteoporosis and spontaneous fractures as well $(8,30,31)$. Here, we report that the OPN mediated gain in OCL functions may be associated with hyperactivation of Erk and Akt.

Collectively, these studies indicate that haploinsufficient loss of $N f 1$ in osteoblasts or its progenitors, results in an increased OPN expression, which in turn leads to gain in OCL functions in vivo. The data suggest that measurement of OPN expression could be used as a biomarker for osteoporosis in the NF1 population. Our studies provide evidence that inhibition of OPN may be useful for the pathologic osseous anomalies observed in patients with NF1.

Acknowledgment. We thank Marilyn Wales for administrative support.

\section{REFERENCES}

1. Bollag G, Clapp DW, Shih S, Adler F, Zhang YY, Thompson P, Lange BJ, Freedman MH, McCormick F, Jacks T, Shannon K 1996 Loss of NF1 results in activation of the Ras signaling pathway and leads to aberrant growth in haematopoietic cells. Nat Genet 12:144-148

2. Largaespada DA, Brannan CI, Jenkins NA, Copeland NG 1996 Nf1 deficiency causes Ras-mediated granulocyte/macrophage colony stimulating factor hypersensitivity and chronic myeloid leukaemia. Nat Genet 12:137-143

3. Yu X, Chen S, Potter OL, Murthy SM, Li J, Pulcini JM, Ohashi N, Winata T, Everett ET, Ingram D, Clapp WD, Hock JM 2005 Neurofibromin and its inactivation of Ras are prerequisites for osteoblast functioning. Bone 36:793-802
4. Crawford AH Jr, Bagamery N 1986 Osseous manifestations of neurofibromatosis in childhood. J Pediatr Orthop 6:72-88

5. Kuorilehto T, Ekholm E, Nissinen M, Hietaniemi K, Hiltunen A, Paavolainen P, Penttinen R, Peltonen J 2006 NF1 gene expression in mouse fracture healing and in experimental rat pseudarthrosis. J Histochem Cytochem 54:363-370

6. Stevenson DA, Moyer-Mileur LJ, Murray M, Slater H, Sheng X, Carey JC, Dube B, Viskochil DH 2007 Bone mineral density in children and adolescents with neurofibromatosis type 1. J Pediatr 150:83-88

7. Illes T, Halmai V, de Jonge T, Dubousset J 2001 Decreased bone mineral density in neurofibromatosis-1 patients with spinal deformities. Osteoporos Int 12:823-827

8. Stevenson DA, Zhou H, Ashrafi S, Messiaen LM, Carey JC, D'Astous JL, Santora SD, Viskochil DH 2006 Double inactivation of NF1 in tibial pseudarthrosis. Am J Hum Genet 79:143-148

9. Teitelbaum SL 2000 Osteoclasts, integrins, and osteoporosis. J Bone Miner Metab 18:344-349

10. Yang FC, Chen S, Robling AG, Yu X, Nebesio TD, Yan J, Morgan T, Li X, Yuan J, Hock J, Ingram DA, Clapp DW 2006 Hyperactivation of p21 and PI3K cooperate to alter murine and human neurofibromatosis type 1-haploinsufficient osteoclast functions. J Clin Invest 116:2880-2891

11. Weber GF, Cantor H 1996 The immunology of Eta-1/osteopontin. Cytokine Growth Factor Rev 7:241-248

12. Liaw L, Birk DE, Ballas CB, Whitsitt JS, Davidson JM, Hogan BL 1998 Altered wound healing in mice lacking a functional osteopontin gene (spp1). J Clin Invest 101:1468-1478

13. Chellaiah MA, Hruska KA 2003 The integrin alpha (v) beta(3) and CD44 regulate the actions of osteopontin on osteoclast motility. Calcif Tissue Int 72:197-205

14. Shapses SA, Cifuentes M, Spevak L, Chowdhury H, Brittingham J, Boskey AL, Denhardt DT 2003 Osteopontin facilitates bone resorption, decreasing bone mineral crystallinity and content during calcium deficiency. Calcif Tissue Int 73:86-92

15. Standal T, Borset M, Sundan A 2004 Role of osteopontin in adhesion, migration, cell survival and bone remodeling. Exp Oncol 26:179-184

16. Chellaiah MA, Kizer N, Biswas R, Alvarez U, Strauss-Schoenberger J, Rifas L, Rittling SR, Denhardt DT, Hruska KA 2003 Osteopontin deficiency produces osteoclast dysfunction due to reduced CD44 surface expression. Mol Biol Cell 14:173-189

17. Yoshitake H, Rittling SR, Denhardt DT, Noda M 1999 Osteopontin-deficient mice are resistant to ovariectomy-induced bone resorption. Proc Natl Acad Sci USA 96:8156-8160

18. Wu X, Estwick SA, Chen S, Yu M, Ming W, Nebesio TD, Li Y, Yuan J, Kapur R, Ingram D, Yoder MC, Yang FC 2006 Neurofibromin plays a critical role in modulating osteoblast differentiation of mesenchymal stem/progenitor cells. Hum Mol Genet 15:2837-2845

19. Hiatt KK, Ingram DA, Zhang Y, Bollag G, Clapp DW 2001 Neurofibromin GTPase-activating protein-related domains restore normal growth in $\mathrm{Nf1}-/-$ cells. J Biol Chem 276:7240-7245

20. Cao JJ, Wronski TJ, Iwaniec U, Phleger L, Kurimoto P, Boudignon B, Halloran BP 2005 Aging increases stromal/osteoblastic cell-induced osteoclastogenesis and alters the osteoclast precursor pool in the mouse. J Bone Miner Res 20:1659-1668

21. Kolanczyk M, Kossler N, Kuhnisch J, Lavitas L, Stricker S, Wilkening U, Manjubala I, Fratzl P, Sporle R, Herrmann BG, Parada LF, Kornak U, Mundlos S 2007 Multiple roles for neurofibromin in skeletal development and growth. Hum Mol Genet 16:874-886

22. Ishii T, Ohshima S, Ishida T, Mima T, Tabunoki Y, Kobayashi H, Maeda M, Uede T, Liaw L, Kinoshita N, Kawase I, Saeki Y 2004 Osteopontin as a positive regulator in the osteoclastogenesis of arthritis. Biochem Biophys Res Commun 316:809-815

23. Sugatani T, Alvarez UM, Hruska KA 2003 Activin A stimulates IkappaB-alpha/ NFkappaB and RANK expression for osteoclast differentiation, but not AKT survival pathway in osteoclast precursors. J Cell Biochem 90:59-67

24. Rangaswami H, Bulbule A, Kundu GC 2005 JNK1 differentially regulates osteopontin-induced nuclear factor-inducing kinase/MEKK1-dependent activating protein-1mediated promatrix metalloproteinase-9 activation. J Biol Chem 280:19381-19392

25. Scherpereel A, Lee YC 2007 Biomarkers for mesothelioma. Curr Opin Pulm Med 13:339-443

26. El-Tanani MK, Campbell FC, Kurisetty V, Jin D, McCann M, Rudland PS 2006 The regulation and role of osteopontin in malignant transformation and cancer. Cytokine Growth Factor Rev 17:463-474

27. Gutmann DH, Wu YL, Hedrick NM, Zhu Y, Guha A, Parada LF 2001 Heterozygosity for the neurofibromatosis 1 (NF1) tumor suppressor results in abnormalities in cell attachment, spreading and motility in astrocytes. Hum Mol Genet 10:3009-3016

28. Gingery A, Bradley E, Shaw A, Oursler MJ 2003 Phosphatidylinositol 3-kinase coordinately activates the MEK/ERK and AKT/NFkappaB pathways to maintain osteoclast survival. J Cell Biochem 89:165-179

29. Abbas S, Clohisy JC, Abu-Amer Y 2003 Mitogen-activated protein (MAP) kinases mediate PMMA-induction of osteoclasts. J Orthop Res 21:1041-1048

30. Digilio MC, Sarkozy A, Capolino R, Chiarini Testa MB, Esposito G, de Zorzi A, Cutrera R, Marino B, Dallapiccola B 2008 Costello syndrome: clinical diagnosis in the first year of life. Eur J Pediatr 167:621-628

31. Hou JW 2008 Rapidly progressive scoliosis after successful treatment for osteopenia in Costello syndrome. Am J Med Genet A 146:393-396 